\title{
Aortic valve reimplantation using Kuban Cuff modification in a patient with acute type A aortic dissection and aortic valve insufficiency
}

\author{
Sergey Boldyrev ${ }^{1,2}$, Vasily Kaleda ${ }^{1}$, Kirill Barbukhatty ${ }^{1,2}$ \\ ${ }^{1}$ Department for Adult Cardiac Surgery, S.V. Ochapowski Regional Hospital \#1, Krasnodar, Russia; ${ }^{2}$ Division for Cardiac Surgery and Cardiology, \\ Kuban State Medical University, Krasnodar, Russia \\ Correspondence to: Sergey Boldyrev. Department for Cardiac Surgery \#2, S.V. Ochapowski Regional Hospital \#1, 140, Rossiyskaya Street, Krasnodar, \\ 350086, Russia. Email: bolsy@rambler.ru.
}

Submitted Apr 27, 2016. Accepted for publication May 09, 2016.

doi: 10.21037/acs.2016.07.03

View this article at: http://dx.doi.org/10.21037/acs.2016.07.03

\section{Clinical vignette}

The corresponding video is an aortic valve (AV) reimplantation using the Kuban Cuff modification technique (Video 1). The patient is a 36-year-old male with acute (3 days) DeBakey type I aortic dissection. He has Marfan syndrome, aortic root aneurysm of $65 \mathrm{~mm}$, tricuspid aortic valve with aortic grade 3 insufficiency. The patient had a previous abdominal aortic replacement without any other significant comorbidities. The left ventricular function and coronary arteries are normal. AV reimplantation was chosen by reason of young age.

\section{Surgical techniques}

\section{Preparation}

Before surgery, all patients with aortic dissection undergo preoperative computed tomography (CT) and echocardiography to assess the details of the patient's anatomy, morphology of the dissection and aortic valve function. The patient is positioned supine and maintained under general anesthesia. A left radial arterial pressure line is placed. A $7 \mathrm{Fr}$ triple-lumen central venous catheter must be inserted into the right jugular vein and a $12 \mathrm{Fr}$ dual-lumen catheter must be inserted into the left subclavian vein. The groins should be exposed in case femoral cannulation is required.

\section{Operation}

Our standard approach for ascending aortic dissection is median sternotomy; artificial circulation is usually performed via cannulation of the innominate artery before pericardiotomy. Operation is routinely performed under moderate hypothermia $\left(28\right.$ to $\left.32{ }^{\circ} \mathrm{C}\right)$; the myocardium protection is conventionally performed using retrograde crystalloid cardioplegia. After the cardioplegia completion, excision of tissues involved in dissection and/or aneurysmatic tissues is performed leaving tissue strips about $4-5 \mathrm{~mm}$ attached to the aortic annulus; the coronary arteries are excised as buttons. Then measurement of the aortic annulus and preparation of the vascular prosthesis for reimplantation are performed. For this manipulation we have developed an original device (Patent No. 2554222 RU 2015) which consists of a cylinder with a truncated cone and a rounded tip of a different diameter: 21, 23, 25, 27, and $29 \mathrm{~mm}$ (1). On the opposite tip of the device there is a circular stop. Onto the working part of each cylinder there are $0.2 \mathrm{~mm}$ deep steam-lined circular furrows at intervals of $2 \mathrm{~mm}$ apart (Figure 1). The aortic annulus is measured by inserting the device into the left ventricle through the aortic annulus, the size of which corresponds to one of the sizes of the device working part. Then the vascular prosthesis for AV reimplantation is prepared. For this purpose we propose a new way of construction of the proximal part of the vascular prosthesis. The vascular prosthesis is selected using the following formula: size of the vascular prosthesis $=$ the size of the chosen device $+8 \mathrm{~mm}$. Then the prosthesis is narrowed proximally to the size of the selected device with two purse-string sutures using polyester threads 2-0, applied horizontally as follows: the assistant inserts the device selected after the aortic annulus measurement into 


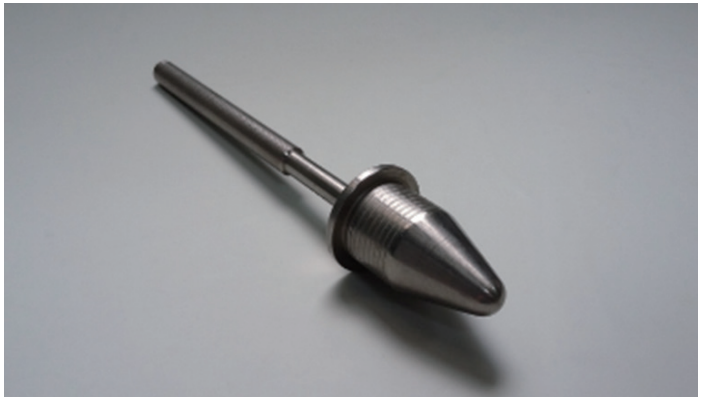

Figure 1 The original device. It has changeable cylinders with different diameters.

the vascular prosthesis selected using the above mentioned formula until the circular stop. The surgeon performs the first horizontal suture. The first suture line is located 1-2 $\mathrm{mm}$ proximal to the prosthesis edge. The suture is tied partially by fixing the prosthesis on one of the antislip circular furrows. The second suture line is located 3-4 mm superior to the first line. At that, the suture edges are arranged checker-wise. Therefore, we obtain an up to $5 \mathrm{~mm}$ wide border (cuff). The cuff diameter corresponds to the aortic annulus size. Then the $U$-shaped sutures on the $3 \times 7 \mathrm{~mm}^{2}$ Teflon liners using the polyester threads are applied inferior to the cusp basis in the transmural direction from inside outward. After the suturing of the aortic annulus, the $\mathrm{AV}$ is reimplanted and fixed inside the vascular prosthesis. To fix the proximal part of the prosthesis more tightly with the polyester threads suturing throughout the proximal part of the vascular prosthesis the prosthesis is fixed alternately in the narrowed part as follows: $1-2 \mathrm{~mm}$ below, between and 1-2 $\mathrm{mm}$ above the cuff projection formed by the two horizontal sutures. Therefore, a zigzagshaped fixation line is formed over the whole circumference (1). The operation is terminated in a typical manner.

\section{Comments}

The AV reimplantation surgery has largely become popular as an alternative to $\mathrm{AV}$ replacement in patients with aneurysm and/or aortic root and ascending aortic dissection (2). The interim results of the reimplantation surgery are promising; the indications for use of this technique are extended for patients with Marfan syndrome, acute Stanford type A aortic dissection, dilatation of the pulmonary autograft after the Ross procedure. The key point of this operation is creation of neosinuses providing long-term satisfactory functioning of the AV. For their creation in the new aortic root different modifications have been proposed but a single consistent surgical approach has not been established yet. Since the first experience of the AV reimplantation technique used (3), multiple modifications of the original method have been proposed. The Seattle modification was one of the first ones proposed which partially resolved the problem of the creation of pseudosinuses. Then, virtually simultaneously, two techniques were proposed: the David IV technique in which the prosthesis formula of aortic annulus $+4 \mathrm{~mm}$ is used and the Miller technique (the vascular prosthesis is 6-8 $\mathrm{mm}$ larger than the aortic annulus which is narrowed on the markedly simplified for the AV with one polyester thread). The latter simplified markedly the operation: the sinuses of Valsalva similar to the natural ones are created; the manipulations inside the prosthesis are performed more easily.

Despite the advantages of the AV reimplantation technique, regarding the valve-related complications such as bleeding, thromboembolism, endocarditis, and patientprosthesis mismatch, the place of such operations in the surgery of dissection has not been determined yet for many reasons. Firstly, dissection is a life-threatening condition due to the aortic rupture risk and malperfusion which lead to the high mortality rate and complications. Secondly, dissection patients' admissions to hospital are often unplanned or occur at nighttime so most surgeons prefer performing an easier technique of the AV resuspension. Thirdly, the aortic root dilatation is often not presented; in this case it is difficult to use the reimplantation technique due to a higher risk. Fourthly, in many hospitals only experienced surgeons perform AV reimplantation.

Our experience of AV reimplantation with mainly Miller and David IV modifications has revealed some challenges such as marked proximal plication of the prosthesis, prosthesis slipping at the moment of narrowing on the usual measurer, one fixation line which in its turn is a weak point regarding the cutting out and bleeding issues. We have faced one complication in the form of spurting bleeding from the area of anastomosis after the restoration of the cardiac function. With some technical difficulties we managed to resolve this problem.

To eliminate the abovementioned difficulties, we have started applying two sutures instead of one, as well as a zigzag-shaped fixation line of the proximal part of the prosthesis to the left ventricular outflow tract. We have also developed a device facilitating the prosthesis plication.

At the beginning of our experience we used plastic aortic 
measurer which contributed to the increased prosthesis slipping as the latter was not fixed during the manipulation. These two insignificant moments, as they might appear at first sight, turned into large inconveniences during long-duration and sometimes dramatic operations. After we had started using our new device we achieved several advantages: the circular stop on the opposite side of the device tip helps to fix the prosthesis and the shallow streamlined circular furrows prevent anterior-posterior slipping on the latter during the fixation of the threads while forming the proximal part.

Three techniques of aortic annuloplasty currently exist and are widely used in ascending aortic aneurysm: suture annuloplasty, internal ring and external ring. Ring annuloplasty is currently more preferable (4). Aortic regurgitation is observed in up to $70 \%$ of patients with acute dissection. But the role of various annuloplasty techniques in case of acute dissection is rarely discussed. We consider that two lines of the sutures for modeling the proximal part of the vascular prosthesis after reimplantation of the latter perform the same functions as the external ring for annuloplasty: the load onto the tissue is reduced and, most importantly, the fibrous ring is stabilized. We finally stopped using a zigzag-shaped line for the prosthesis fixation since we had started using only three sutures for the prosthesis fixation (5). In case of acute aortic dissection and often a worrying reconstruction of the aortic root the duration of the intervention was on the critical path. Using our technique it is not necessary to reduce the ring additionally in the presence of widening of the latter: a pre-prepared vascular prosthesis is a priori a little smaller than the external diameter of the root, hence, in any case enlargement of the coaptation zone of the AV cusps occurs. Moreover, the proximal part shape obtained is no worse than the "collar" of the Valsalva Graft. Our cuff allows aligning the irregularity of the aortic root separation from the surrounding tissues, particularly, in the area of the right coronary cusp attachment, the interventricular septum on the level of the sinuses lower points.

\section{Acknowledgements}

None.

\section{Footnote}

Conflicts of Interest: The authors have no conflicts of interest to declare.

\section{References}

1. Boldyrev S, Barbukhatty K, Porhanov V. A Novel Tool to Facilitate Crimping Suture Placement for a Modified David V/Miller Aortic Root Replacement. Aorta (Stamford) 2014;2:161-6.

2. Franke UF, Isecke A, Nagib R, et al. Quality of life after aortic root surgery: reimplantation technique versus composite replacement. Ann Thorac Surg 2010;90:1869-75.

3. David TE, Feindel CM. An aortic valve-sparing operation for patients with aortic incompetence and aneurysm of the ascending aorta. J Thorac Cardiovasc Surg 1992;103:61721; discussion 622.

4. de Kerchove L, Vismara R, Mangini A, et al. In vitro comparison of three techniques for ventriculoaortic junction annuloplasty. Eur J Cardiothorac Surg 2012;41:1117-23; discussion 1123-4.

5. Cameron D, Vricella L. Valvesparing aortic root replacement with the valsalva graft. OpTech Thorac Cardiovasc Surg 2009;14:297-308.
Cite this article as: Boldyrev S, Kaleda V, Barbukhatty K. Aortic valve reimplantation using Kuban Cuff modification in a patient with acute type A aortic dissection and aortic valve insufficiency. Ann Cardiothorac Surg 2016;5(4):404-406. doi: 10.21037/acs.2016.07.03 